\title{
Gynecologic and Obstetric Implications of Darier Disease: A Dermatologist's Perspective
}

\author{
Naveen Kumar Kansal, MD
}

\section{PRACTICE POINTS}

- Because Darier disease (DD) manifests during reproductive years, systemic retinoids should be used carefully in female patients.

- For a Papanicolaou test to be properly interpreted in a patient with $\mathrm{DD}$, the cytopathologist must be informed of the DD diagnosis.

- Darier disease may be exacerbated or relieved during pregnancy.

$\mathrm{D}$ arier disease (DD) (also known as dyskeratosis follicularis) is a rare, autosomal-dominant genodermatosis characterized by greasy, rough, keratotic papules; typical nail abnormalities; mucosal changes; and characteristic dyskeratotic acantholysis that is called corps ronds and grains on histopathologic analysis. Darier disease is caused by mutations of the ATP2A2 gene on chromosome 12q23-24. ${ }^{1,2}$

Because of the autosomal-dominant pattern of inheritance in $\mathrm{DD}$, if either parent is affected by DD, approximately $50 \%$ of their offspring will have the disorder. Therefore, couples need to be offered genetic counseling at a preconception visit or early in pregnancy. Although penetrance of DD is complete, spontaneous mutations are frequent and expressivity is variable ${ }^{1}$; prenatal diagnosis, though available since the 1980s, is therefore unreliable in $\mathrm{DD}$, given the considerable variation in phenotypic expressivity. Differing phenotypes underscore the importance of proper counseling by the treating dermatologist or other provider. Females with a mild or nearly undetectable phenotype can give birth to a child with severe disease.

Lack of clear understanding about the variable phenotypic expressivity of DD can cause considerable anger, anxiety, guilt, psychological trauma, and fear in parents, should their child later develop a severe phenotype. They may feel that they were not properly prepared for the outcome. The physician-parent or physician-patient relationship can be negatively impacted if ongoing counseling is inadequate.

Clinically, DD presents in early adolescence (age range, 6-20 years) in most patients, which means that the disease and female reproductive years are contemporaneous. However, gynecologic and obstetric issues and complications of DD rarely have been addressed. ${ }^{3}$ Oromucosal involvement in DD is reported in 13\% to $50 \%$ of cases, yet vaginal and cervical mucosal involvement rarely has been described, ${ }^{4,5}$ likely due to underreporting. Therefore, in this rare disease, it is important to address these aspects so that the patients are provided with appropriate management options.

\section{Implications for Cervical Screening and Papanicolaou Tests}

Cytopathologic findings of a Papanicolaou test taken from a patient with DD can lead to erroneous diagnosis of a low-grade squamous intraepithelial lesion due to cervical involvement by the disease process; therefore, correct interpretation of a smear may be inappropriate and erroneous. The cytopathologist needs to be informed of the patient's diagnosis of DD in advance for appropriate reporting. 5,6

From the Department of Dermatology and Venereology, All India Institute of Medical Sciences, Rishikesh.

The author reports no conflict of interest.

Correspondence: Naveen Kumar Kansal, MD, Department of Dermatology and Venereology, All India Institute of Medical Sciences,

Rishikesh 249 203, India (kansalnaveen29@gmail.com).

doi:10.12788/cutis.0222 


\section{Obstetric Implications}

Fertility is normal in DD patients, and pregnancy usually has a normal course; however, exacerbation and remission of disease have been reported. de la Rosa Carrillo ${ }^{7}$ reported a case of vegetating DD during pregnancy. He described it as an exacerbation with concurrent bacterial infection and bilateral external otitis. ${ }^{7}$ Spouge et $\mathrm{al}^{8}$ reported a case of a 58 -year-old woman who was the mother of 4 DD patients. She experienced an exacerbation of DD during all 6 pregnancies but improved immediately postpartum. ${ }^{8}$ Espy et al ${ }^{9}$ evaluated 8 cases of women with DD and described spontaneous improvement of the disorder during pregnancy ( 1 case) or while taking an oral contraceptive (3 cases).

\section{Prenatal Counseling}

Women with DD should be encouraged to talk to their dermatologist, obstetrician, or other provider of prenatal care regarding plans for pregnancy, labor, and delivery, as these events might be affected by the disorder. During pregnancy, careful monitoring and self-care remain essential. Simple measures to reduce the impact of irritants on DD during pregnancy include keeping the skin cool, using a soothing moisturizer, applying photoprotection, and using sunscreen. Treatment with systemic retinoids must be avoided if pregnancy is planned.

Warty plaques and papules of DD can involve flexures (groin, vulva, and perineum), with resultant malodor and pruritus ${ }^{10}$ as well as the potential for (drug resistant) secondary infection (eg, Staphylococcus aureus, group B Streptococcus, viruses [eg, Kaposi varicelliform eruption]). Skin swabs should be taken for culture and susceptibility testing, and infection should be treated at the earliest sign.

\section{Management Concerns During Pregnancy and Delivery}

Because the benefits of treating DD might outweigh risk in certain cases, thorough discussion with the patient about options is recommended, including the following concerns:

- Because mucocutaneous elasticity of the birth canal, including the vulva, perineum, and groin, is essential for nontraumatic vaginal delivery, it might be necessary to schedule an elective cesarean delivery in DD patients in whom these regions are involved. ${ }^{11}$

- In females with lower abdominal lesions, using a Pfannenstiel-Kerr incision for cesarean delivery might be problematic. ${ }^{11}$

- A single case report has described successful anesthetic management of labor, delivery, and postpartum care in a DD patient. ${ }^{12}$ Involvement of the skin of the back might preclude safe administration of regional anesthesia; however, because DD lesions are considered noninfectious, the authors operatively administered a subarachnoid block at the L3-L4 interspace through a lesion-free area. Postpartum, the patient was observed in the intensive care unit. She and the baby remained stable; she did not develop infectious complications, including a central nervous system infection. ${ }^{12}$
- Mucosal involvement is relatively rare in DD and has not been reported to compromise airway management. ${ }^{8}$

\section{Postnatal Considerations}

Breastfeeding might have to be stopped early or withheld altogether if there is widespread involvement of the skin of the breast or the nipple. ${ }^{11}$ Darier disease has been associated with neuropsychiatric manifestations, including major depression $(30 \%)$, suicide attempts $(13 \%)$, suicidal thoughts $(31 \%)$, cyclothymia, bipolar disorder $(4 \%)$, and epilepsy (3\%). ${ }^{13,14}$ Therefore, patients should be screened for postpartum psychiatric manifestations at an early follow-up visit.

\section{Final Thoughts}

Although the etiology of DD is well known, the gynelogic and obstretric implications of this genodermatosis have rarely been described. This brief commentary is an attempt to provide the important information to a practicing dermatologist for appropriate management of female DD patients.

\section{REFERENCES}

1. Bale SJ, Toro JR. Genetic basis of Darier-White disease: bad pumps cause bumps. J Cutan Med Surg. 2000;4:103-106. doi:10.1177/120347540000400212

2. Kansal NK, Hazarika N, Rao S. Familial case of Darier disease with guttate leukoderma: a case series from India. Indian Dermatol Online J. 2018;9:62-63. doi:10.4103/idoj.IDOJ_52_17

3. Lynch PJ. Vulvar dermatoses: the eczematous diseases. In: Black M, Ambros-Rudolph CM, Edwards L, Lynch P, eds. Obstetric and Gynecologic Dermatology. 3rd ed. Mosby-Elsevier; 2008:192-194.

4. Adam AE. Ectopic Darier's disease of the cervix: an extraordinary cause of an abnormal smear. Cytopathology. 1996;7:414-421. doi:10.1111/j.1365-2303.1996.tb00547.x

5. Suárez-Peñaranda JM, Antúnez JR, Del Rio E, et al.Vaginal involvement in a woman with Darier's disease: a case report. Acta Cytol. 2005;49:530532. doi:10.1159/000326200

6. Boon ME. Dr. Darier's lesson: it can be advantageous to the patient to ignore evident cytonuclear changes. Acta Cytol. 2005;49:469470. doi:10.1159/000326189

7. de la Rosa Carrillo D.Vegetating Darier's disease during pregnancy. Acta Derm Venereol. 2006;86:259-260. doi:10.2340/00015555-0066

8. Spouge JD, Trott JR, Chesko G. Darier-White's disease: a cause of white lesions of the mucosa. report of four cases. Oral Surg Oral Med Oral Pathol. 1966;21:441-457. doi:10.1016/00304220(66)90401-4

9. Espy PD, Stone S, Jolly HW Jr. Hormonal dependency in Darier disease. Cutis. 1976;17:315-320.

10. De D, Kanwar AJ, Saikia UN. Uncommon flexural presentation of Darier disease. J Cutan Med Surg. 2008;12:249-252. doi:10.2310/7750.2008.07035

11. Quinlivan JA, O'Halloran LC. Darier's disease and pregnancy. Dermatol Aspects. 2013;1:1-3. doi:10.7243/2053-5309-1-1

12. Sharma R, Singh BP, Das SN. Anesthetic management of cesarean section in a parturient with Darier's disease. Acta Anaesthesiol Taiwan. 2010;48:158-159. doi:10.1016/S1875-4597(10)60051-3

13. Gordon-Smith K, Jones LA, Burge SM, et al. The neuropsychiatric phenotype in Darier disease. Br J Dermatol. 2010;163:515-522. doi:10.1111/j.1365-2133.2010.09834.x

14. Dodiuk-Gad RP, Cohen-Barak E, Khayat M, et al. Darier disease in Israel: combined evaluation of genetic and neuropsychiatric aspects. Br J Dermatol. 2016;174:562-568. doi:10.1111/ bjd. 14220 